\title{
Erratum for: A study of homogeneity in relational databases [Annals of Mathematics and Artificial Intelligence 33(2) (2001) 379-414]
}

\author{
José Maria Turull Torres \\ Massey University, Information Science Research Centre, Department of Information Systems, \\ PO Box 756, Wellington, New Zealand \\ E-mail: j.m.turull@massey.ac.nz
}

Keywords: query languages, database machines, query computability, completeness of models

In definition 3.5 in [3], where we define the class $\mathcal{Q C} \mathcal{Q}^{k}$, for every $k \geqslant 1$, we need to add a second requirement for a query to be in the class $\mathcal{Q C} \mathcal{Q}^{k}$, namely that the answer to every query $f$ in the class $\mathcal{Q C} \mathcal{Q}^{k}$ is always the union of complete $F O^{k}$ types. Previously, we considered that property to be implied by our definition, but it turns out that it is not so. There are total computable queries which for some $k \geqslant 1$ preserve realization of $F O^{k}$ types for $k$-tuples, but whose evaluation on some databases defines a relation which has not complete $F O^{k}$ types for $k$-tuples. One example of such queries [1] is the query which defines a total order in the class of Odd Multipedes of Gurevich and Shellah [2].

We give below the correct definition, which should replace definition 3.5 in [3].

\section{Definition 3.5.}

- Let $1 \leqslant r \leqslant k$. A total computable $r$-ary query $f$ is in the class $\mathcal{Q C} \mathcal{Q}^{k}$, if $f$ preserves realization of $F O^{k}$ types for $k$-tuples and if for all databases $I$ in $\mathcal{B}_{\sigma}$, and for all tuples $\bar{a}, \bar{b}$ in $\operatorname{dom}(I)^{r}$, if $\bar{a} \in f(I)$ and $\bar{a} \equiv_{k} \bar{b}$, then also $\bar{b} \in f(I)$.

- $\mathcal{Q} \mathcal{C} \mathcal{Q}^{\omega}=\bigcup_{k \in \omega} \mathcal{Q C} \mathcal{Q}^{k}$.

Correspondingly, definition 5.1 should also be changed in the same way:

Definition 5.1. For any schema $\sigma$, let us denote as $\operatorname{arity}(\sigma)$ the maximum arity of a relation symbol in the schema. We define the class $\mathcal{Q C Q}$ as the class of queries $f \in \operatorname{total}(\mathcal{C Q})$, of some schema $\sigma$ and of any arity, for which there exists an integer $n \geqslant \max \{\operatorname{arity}(f)$, arity $(\sigma)\}$, such that for every pair of databases $I, J$ in $\mathcal{B}_{\sigma}$, if $I \equiv_{\tau_{h}} J$, then $\langle I, f(I)\rangle \equiv_{\tau_{h}}\langle J, f(J)\rangle$, where $h=\max \{n, \min \{k: I$ and $J$ are strongly $k$-homogeneous $\}$ \}. We also require that the answer to the query $f$ must be the union of complete $F O^{h}$ types, i.e., for all databases $I$ in $\mathcal{B}_{\sigma}$, and for all tuples $\bar{a}, \bar{b}$ in $\operatorname{dom}(I)^{r}$, if 
$\bar{a} \in f(I)$ and $t p_{I}^{F O^{h}}(\bar{a})=t p_{I}^{F O^{h}}(\bar{b})$, then also $\bar{b} \in f(I)$. As in definition 3.4, regarding the last equivalence we consider the schema $\sigma \cup\{f\}$, where $f$ is a relation symbol with the arity of the query.

\section{Acknowledgements}

I am deeply grateful to an anonymous referee who after recently reviewing a submission where I used the same strategy with a different logic, pointed me out this mistake. I am also grateful to Anuj Dawar and Lauri Hella, for their helpful assistance in finding a counterexample.

\section{References}

[1] A. Dawar, Personal communication (2003).

[2] Y. Gurevich and S. Shelah, On finite rigid structures, Journal of Symbolic Logic 61 (1996).

[3] J.M. Turull Torres, A study of homogeneity in relational databases, Annals of Mathematics and Artificial Intelligence 33(2) (2001) 379-414. 